\title{
Phase I study of docetaxel plus $S-1$ combination chemotherapy for recurrent non-small cell lung cancer
}

\author{
KAZUNORI FUJITAKA, NOBORU HATTORI, TADASHI SENOO, HIROSHI IWAMOTO, \\ SHINICHIRO OHSHIMO, MASASHI KANEHARA, NOBUHISA ISHIKAWA, \\ YOSHINORI HARUTA, HIROSHI MURAI and NOBUOKI KOHNO \\ Department of Molecular and Internal Medicine, Graduate School of Biomedical Science, \\ Hiroshima University, Hiroshima 734-8551, Japan
}

Received August 6, 2010; Accepted November 11, 2010

DOI: $10.3892 / 01.2010 .218$

\begin{abstract}
S-1 is a novel oral fluorouracil prodrug that plays a role in non-small cell lung cancer (NSCLC). Docetaxel (DTX) is one of the standard agents for relapsed NSCLC. We performed a phase I study of DTX plus S-1 combination therapy as second-line treatment for NSCLC to determine the maximum tolerated dose (MTD) and recommended dose (RD). Patients with recurrent NSCLC, aged 20-74 years with an Eastern Cooperative Oncology Group performance status of 0-1 and measurable lesions, were enrolled. The treatment consisted of four dose levels. The patients received DTX (40-60 mg/m² intravenously on day 1) and S-1 (65-80 mg/ $\mathrm{m}^{2}$ orally, daily on days $1-14$ ) for each 21 -day cycle. Three to six patients were treated at each dose level with the two drugs, with MTD defined as the dose level at which doselimiting toxicity (DLT) occurred in $33 \%$ of the patients. A total of 17 patients were enrolled. At dose level 4 (DTX, 60 $\left.\mathrm{mg} / \mathrm{m}^{2} ; \mathrm{S}-1,80 \mathrm{mg} / \mathrm{m}^{2}\right) 3$ of 5 patients experienced DLT and this level was regarded as the MTD. Therefore, dose level 3 (DTX, $60 \mathrm{mg} / \mathrm{m}^{2} ; \mathrm{S}-1,65 \mathrm{mg} / \mathrm{m}^{2}$ ) was selected as the RD for subsequent studies. The DLTs were neutropenia (grade 4) and mucositis (grade 3). The response rate was 5.9\% (1 of 17 patients achieved a partial response) and 14 of 17 patients achieved stable disease. This combination regimen showed a tolerable and manageable profile in recurrent NSCLC and therefore warrants further evaluation.
\end{abstract}

\section{Introduction}

Lung cancer is the leading cause of cancer-related mortality in Japan, and other countries. A meta-analysis of randomized controlled trials has shown that platinum-based chemotherapy

Correspondence to: Dr Noboru Hattori, Department of Molecular and Internal Medicine, Graduate School of Biomedical Science, Hiroshima University, 1-2-3 Kasumi, Minami-ku, Hiroshima 734-8551, Japan

E-mail: nhattori@hiroshima-u.ac.jp

Key words: docetaxel, S-1, phase I prolongs survival time in advanced non-small cell lung cancer (NSCLC) (1). However, the majority of patients with advanced NSCLC progress or relapse after receiving first-line platinumbased chemotherapy. While advanced NSCLC has a poor prognosis, second-line chemotherapy remains a key component in the clinical management of NSCLC patients.

Docetaxel (DTX) is a taxane originally extracted from the needles of the European yew tree Taxus baccata. DTX exerts its antitumor activity through the acceleration of microtubule assembly from tubulin subunits and the inhibition of microtubule depolymerization. DTX has been shown to improve the survival and quality of life in patients with NSCLC previously treated with platinum-based therapy compared to best supportive care (2). DTX is used as standard second-line chemotherapy for advanced NSCLC in a similar manner as other agents, such as pemetrexed and erlotinib.

$\mathrm{S}-1$ is an oral fluoropyrimidine comprising tegafur, 5-chloro2,4-dihydroxypyridine (CDHP) and potassium oxonate (OXO) (3). Tegafur is a prodrug of 5-fluorouracil (5-FU). CDHP increases 5-FU concentration in serum and tumor tissue by inhibiting dihydropyrimidine dehydrogenase (DPD). OXO is included in S-1 in order to reduce tegafur-associated gastrointestinal toxicity (4). 5-FU is not effective for lung cancer, as NSCLC tissue generally has high DPD activity (5). By contrast, S-1 showed antitumor activity in NSCLC. In a phase II study, S-1 monotherapy resulted in a $22 \%$ response rate in patients with advanced chemotherapy-naïve NSCLC, with a median survival time of 10.2 months (6).

DTX and S-1 possess different antitumor mechanisms, and a synergistic effect between the two agents was demonstrated in human breast cancer xenograft models (7). Furthermore, phase I/II studies in advanced gastric cancer showed that this combination was active and well tolerated $(8,9)$.

To further improve the efficacy of second-line chemotherapy for NSCLC, this phase I study was designed in order to evaluate DTX plus S-1 combination therapy.

\section{Patients and methods}

Eligibility. Patients with recurrent NSCLC were eligible for this study. The study was initiated following approval from the institutional review board. The inclusion criteria were: 
i) histologically or cytologically proven NSCLC, ii) measurable or evaluable lesions, iii) prior administration of first-line platinum-based chemotherapy, with or without second-line gefitinib, and iv) an interval of at least 4 weeks following the last administration of prior chemotherapy. Patient characteristics included: i) age 20-74 years, ii) an Eastern Cooperative Oncology Group (ECOG) performance status of 0 or 1 , iii) adequate hematopoietic function (white blood cells, $\geq 4,000 / \mathrm{mm}^{3}$; neutrocytes, $\geq 2,000 / \mathrm{mm}^{3}$; platelets, $\geq 10 \times 10^{4} / \mathrm{mm}^{3}$ and hemoglobin, $\geq 9.5 \mathrm{~g} / \mathrm{dl}$ ), iv) adequate hepatic function (total bilirubin $\leq 1.5 \mathrm{mg} / \mathrm{dl}$ and transaminase $\leq 2$ times institutional normal upper limit), v) serum creatinine $\leq 1.5 \mathrm{mg} / \mathrm{dl}$, vi) adequate pulmonary function $\left(\mathrm{PaO}_{2}>60\right.$ Torr or $\mathrm{SpO}_{2}>90 \%$ ), vii) adequate cardiac function without treatment, viii) anticipated survival $\geq 12$ weeks, and ix) written informed consent prior to treatment. Exclusion criteria were: i) massive plural effusion or pericardial effusion, ii) superior vena cava syndrome, iii) serious concomitant systemic disorders, including active infection, uncontrollable diabetes mellitus, hypertension, uncontrollable angina, severe heart failure, active ulcer or another primary malignancy, iv) active interstitial pneumonia, v) coagulation system disorder, vi) history of drug allergy, including allergy to polysorbate 80 , vii) pregnancy or breastfeeding, and viii) other concomitant anticancer drug administration, including flucytosine.

Treatment schedule and assessment. The patients received variable doses of DTX administered as a 1-h intravenous infusion on day 1 and S-1 administered orally on days 1-14 every 3 weeks. The dose-escalation levels of the two agents are shown in Table I. The starting dose of DTX (dose level 1) was $40 \mathrm{mg} / \mathrm{m}^{2}$. Subsequent dose levels were $50 \mathrm{mg} / \mathrm{m}^{2}$ (dose level 2) and $60 \mathrm{mg} / \mathrm{m}^{2}$ (dose levels 3 and 4). The starting dose of S-1 (dose levels 1-3) was $65 \mathrm{mg} / \mathrm{m}^{2}$, administered according to body surface area (BSA): BSA $<1.25 \mathrm{~m}^{2}, 50 \mathrm{mg} /$ day; $B S A \geq 1.25$ to $<1.50 \mathrm{~m}^{2}, 80 \mathrm{mg} /$ day; $B S A \geq 1.50 \mathrm{~m}^{2}$, $100 \mathrm{mg} /$ day). Subsequent dose levels were $85 \mathrm{mg} / \mathrm{m}^{2}$ (dose level 4), administered according to BSA: BSA $<1.25 \mathrm{~m}^{2}$, $80 \mathrm{mg} /$ day; $B S A \geq 1.25$ to $<1.50 \mathrm{~m}^{2}, 100 \mathrm{mg} /$ day; and BSA $\geq 1.50 \mathrm{~m}^{2}, 120 \mathrm{mg} /$ day).

Toxicity was evaluated according to the National Cancer Institute Common Toxicity Criteria (NCI-CTC), version 2.0. The dose-limiting toxicity (DLT) was defined as: i) grade 4 neutropenia during administration of S-1 or lasting longer than 4 days following S-1 administration, ii) grade 3 neutropenia with fever, iii) grade 4 thrombocytopenia, iv) grade 3-4 non-hematological toxicity other than nausea, vomiting or fatigue, or v) delay of second cycle within 2 weeks. DLT was determined during the first treatment cycle. At least 3 patients at each dose level were enrolled; the criteria were: i) the dose was defined as the maximum tolerated dose (MTD) when all 3 patients developed DLT; ii) when 1-2 of 3 patients developed a DLT, 3 additional patients were enrolled; iii) when $>3$ of 6 patients developed a DLT, the dose was defined as the MTD; and iv) when $<2$ of 6 patients developed a DLT, the dose was increased to the following level. The recommended dose (RD) for the phase II study was defined as the dose preceding the MTD. Treatment continued unless the disease progressed, unacceptable toxicity occurred or the patient refused further treatment. The response to treatment was assessed according
Table I. Dose escalation of the two agents.

\begin{tabular}{lcc}
\hline Level & Docetaxel $\left(\mathrm{mg} / \mathrm{m}^{2}\right)$ & $\mathrm{S}-1\left(\mathrm{mg} / \mathrm{m}^{2}\right)$ \\
\hline 1 & 40 & 65 \\
2 & 50 & 65 \\
3 & 60 & 65 \\
4 & 60 & 80 \\
\hline
\end{tabular}

Table II. Patient characteristics $(n=17)$.

\begin{tabular}{lc}
\hline Characteristics & No. \\
\hline Age (years) & 61 (50-72) \\
Median (range) & \\
Gender & $12 / 5$ \\
Male/Female & \\
PS (ECOG) & $13 / 4$ \\
0/1 & \\
Histology & $10 / 3 / 3 / 1$ \\
Ad/Sq/La/unclassified & \\
Prior chemotherapy & 13 \\
CBDCA + PTX & 3 \\
CDDP + VNR & 1 \\
CBDCA + VP-16 & 4 \\
Gefitinib (additional) & \\
\hline
\end{tabular}

Eastern Cooperative Oncology Group, ECOG; PS, performance status; Ad, adenocarcinoma; Sq, squamous cell carcinoma; La, large cell carcinoma; CBDCA, carboplatin; PTX, paclitaxel; CDDP, cisplatin; VNR, vinorelbine.

to the WHO criteria [response evaluation criteria in solid tumors (RECIST)].

\section{Results}

Patient characteristics. A total of 17 patients were enrolled. Patient characteristics are shown in Table II. The median age at entry was 61 years (range 50-72). A total of 13 patients had a performance status (PS) of 0 and 4 patients had a PS of 1 . The predominant histology was adenocarcinoma. All of the patients had previously been treated with platinum-based chemotherapy and 4 patients had also received gefitinib.

Dose escalation and determination of the maximum tolerated and recommended doses. A total of 3 patients were enrolled at dose levels 1 and 2 and completed the first cycle without DLTs. A total of 6 patients were enrolled at dose level 3 , since 1 of the first 3 patients experienced grade 4 neutropenia. Grade 3 oral mucositis occurred in 1 of the 3 added patients at dose level 3; thus, 4 of 6 patients experienced no DLTs, allowing progression to dose level 4. At dose level 4, 3 of 5 patients developed grade 4 neutropenia. The MTD was therefore defined as dose level 4, and dose level 3 was selected as the RD. 
Table III. Hematological toxicity.

\begin{tabular}{|c|c|c|c|c|c|c|c|c|c|c|c|c|c|c|c|c|}
\hline \multirow[t]{2}{*}{ NCI-CTC grade } & \multicolumn{4}{|c|}{ Level 1} & \multicolumn{4}{|c|}{ Level 2} & \multicolumn{4}{|c|}{ Level 3} & \multicolumn{4}{|c|}{ Level 4} \\
\hline & 1 & 2 & 3 & 4 & 1 & 2 & 3 & 4 & 1 & 2 & 3 & 4 & 1 & 2 & 3 & 4 \\
\hline Leukopenia & 0 & 0 & 0 & 0 & 1 & 0 & 1 & 0 & 0 & 3 & 1 & 0 & 0 & 2 & 3 & 0 \\
\hline Neutropenia & 1 & 0 & 1 & 0 & 1 & 1 & 1 & 0 & 0 & 1 & 2 & 1 & 0 & 1 & 1 & 3 \\
\hline Anemia & 0 & 0 & 0 & 0 & 0 & 1 & 0 & 0 & 2 & 2 & 0 & 0 & 1 & 1 & 0 & 0 \\
\hline Thrombocytopenia & 0 & 0 & 0 & 0 & 0 & 0 & 0 & 0 & 0 & 0 & 0 & 0 & 0 & 0 & 0 & 0 \\
\hline $\begin{array}{l}\text { Blood bilirubin } \\
\text { increased }\end{array}$ & 0 & 0 & 0 & 0 & 0 & 0 & 0 & 0 & 1 & 0 & 0 & 0 & 0 & 0 & 0 & 0 \\
\hline Hypoalbuminemia & 0 & 0 & 0 & 0 & 0 & 0 & 0 & 0 & 0 & 1 & 0 & 0 & 1 & 0 & 0 & 0 \\
\hline
\end{tabular}

NCI-CTC, National Cancer Institute Common Toxicity Criteria.

Table IV. Non-hematological toxicity.

\begin{tabular}{|c|c|c|c|c|c|c|c|c|c|c|c|c|c|c|c|c|}
\hline \multirow[t]{2}{*}{ NCI-CTC grade } & \multicolumn{4}{|c|}{ Level 1} & \multicolumn{4}{|c|}{ Level 2} & \multicolumn{4}{|c|}{ Level 3} & \multicolumn{4}{|c|}{ Level 4} \\
\hline & 1 & 2 & 3 & 4 & 1 & 2 & 3 & 4 & 1 & 2 & 3 & 4 & 1 & 2 & 3 & 4 \\
\hline Fatigue & 1 & 0 & 0 & 0 & 1 & 0 & 0 & 0 & 1 & 0 & 0 & 0 & 2 & 0 & 0 & 0 \\
\hline Appetite loss & 1 & 0 & 0 & 0 & 2 & 0 & 0 & 0 & 1 & 2 & 0 & 0 & 2 & 0 & 0 & 0 \\
\hline Nausea & 1 & 0 & 0 & 0 & 1 & 0 & 0 & 0 & 1 & 0 & 0 & 0 & 0 & 0 & 0 & 0 \\
\hline Diarrhea & 0 & 0 & 0 & 0 & 0 & 0 & 0 & 0 & 0 & 3 & 0 & 0 & 1 & 0 & 0 & 0 \\
\hline Hiccup & 0 & 1 & 0 & 0 & 0 & 0 & 0 & 0 & 0 & 0 & 0 & 0 & 0 & 0 & 0 & 0 \\
\hline Mucositis & 0 & 0 & 0 & 0 & 1 & 0 & 0 & 0 & 0 & 0 & 1 & 0 & 1 & 0 & 0 & 0 \\
\hline Alopecia & 1 & 0 & 0 & 0 & 1 & 1 & 0 & 0 & 0 & 0 & 0 & 0 & 1 & 1 & 0 & 0 \\
\hline Hypersensitivity & 0 & 0 & 0 & 0 & 1 & 0 & 0 & 0 & 0 & 0 & 0 & 0 & 0 & 0 & 0 & 0 \\
\hline Muscle pain & 0 & 0 & 0 & 0 & 0 & 0 & 0 & 0 & 1 & 0 & 0 & 0 & 1 & 0 & 0 & 0 \\
\hline Arthlargia & 0 & 0 & 0 & 0 & 0 & 0 & 0 & 0 & 0 & 0 & 0 & 0 & 1 & 0 & 0 & 0 \\
\hline Skin pigmentation & 0 & 0 & 0 & 0 & 0 & 0 & 0 & 0 & 0 & 0 & 0 & 0 & 1 & 0 & 0 & 0 \\
\hline
\end{tabular}

NCI-CTC, National Cancer Institute Common Toxicity Criteria.

Toxicities and response to therapy. Hematological and nonhematological toxicities observed during the first cycle are shown in Tables III and IV. All the toxicities were mild. No grade $3 / 4$ anemia or thrombocytopenia was observed in this study. With respect to non-hematological toxicity, grade 2 diarrhea and appetite loss were observed at dose level 3 .

The patients were able to be evaluated for response. A partial response was observed in 1 patient (5.9\%), while stable disease was observed in 14 patients (82.4\%) and progressive disease occurred in 2 patients (11.7\%). Thus, the overall response rate was $5.9 \%$ ( 1 of 17 patients).

\section{Discussion}

In Japan, molecularly targeted therapies, such as gefitinib, erlotinib and bevacizumab, are used in the clinic and have gradually prolonged the survival of NSCLC patients. However, lung cancer remains the leading cause of cancer-related mortality. Following first-line chemotherapy, the majority of patients with advanced NSCLC progress or relapse and their disease becomes resistant to the cytotoxic agents. According to the American Society of Clinical Oncology (ASCO) guidelines published in 2003 (10), DTX is recommended as a second-line therapy for patients with recurrent NSCLC previously treated with platinum-based therapy. Currently, pemetrexed and erlotinib are also considered to be standard second-line therapies (11).

To improve the efficacy of chemotherapy for NSCLC patients previously treated with platinum-based chemotherapy, certain studies have evaluated DTX-based combination therapy. DTX plus gemcitabine has been reported to yield a response rate of $7 \%$, while DTX plus irinotecan results in a 10-20\% response rate (12). However, no survival differences have been noted between combination chemotherapy and monotherapy in the second-line setting. In the present study, only 1 of 17 patients achieved a partial response, yielding a response rate of $5.9 \%$. An additional 15 patients achieved stable disease. 
Combination treatment with DTX and S-1 has been intensively investigated in gastric cancer. Studies have shown that no pharmacokinetic interactions occur between DTX and S-1 in patients with normal renal function. Impaired renal function often induces severe adverse reactions in patients treated with S-1, since elevated CDHP levels result in higher 5-FU concentrations (13). DTX and S-1 exhibit different antitumor mechanisms, and a synergistic effect between DTX and S-1 has been shown in human breast cancer xenograft models (7). Phase I/II studies in advanced gastric cancer showed that DTX and S-1 combination therapy is active and well tolerated $(8,9)$. In the majority of studies, DTX is administered at $40 \mathrm{mg} / \mathrm{m}^{2}$. A few phase I/II studies have already been performed in relapsed NSCLC (14), in which an identical dosage of DTX was used. However, the present study identified a DTX dose of $60 \mathrm{mg} / \mathrm{m}^{2}$ every 3 weeks as the RD, which is commonly used as standard monotherapy in Japan. Consequently, the RD of the present combination regimen appears to enhance the antitumor effects of S-1 when added to DTX monotherapy.

In the present study, the DLTs were grade 4 neutropenia and grade 3 mucositis. The major hematological toxicities were neutropenia and mild anemia, while the non-hematological toxicities were primarily gastrointestinal toxicities, including nausea, diarrhea and appetite loss. These toxicities were generally well tolerated.

Although other agents, such as EGFR-TKIs, are utilized depending on the EGFR mutation status, DTX monotherapy remains a crucial second-line chemotherapy for advanced NSCLC. Recent studies have shown that sequential or maintenance chemotherapy is useful as a second-line therapy for NSCLC patients who do not present with progressive disease (15). It is crucial to clarify the optimal duration and timing of second-line chemotherapy.

In conclusion, the present study has shown that the combination of DTX and S-1 may be useful and well tolerated as a second-line chemotherapy for NSCLC. Additional studies are required to confirm the objective response rate and survival benefit provided by this combination regimen at the RD determined in this study.

\section{References}

1. Non-small Cell Lung Cancer Collaborative Group: Chemotherapy in non-small cell lung cancer: a meta-analysis using updated data on individual patients from 52 randomised clinical trials. BMJ 311: 899-909, 1995.
2. Shepherd FA, Dancey J, Ramlau R, et al: Prospective randomized trial of docetaxel versus best supportive care in patients with non-small-cell lung cancer previously treated with platinumbased chemotherapy. J Clin Oncol 18: 2095-2103, 2000.

3. Shirasaka T, Shimamato Y, Ohshimo H, et al: Development of a novel form of an oral 5-fluorourcil derivative (S-1) directed to the potentiation of the tumor selective cytotoxicity of 5-fluorouracil by two biochemical modulators. Anticancer Drugs 7: 548-557, 1996.

4. Shirasaka T, Shimamoto Y and Fukushima M: Inhibition by oxonic acid of gastrointestinal toxicity of 5-fluorouracil without loss of its antitumor activity in rats. Cancer Res 53: 4004-4009, 1993.

5. Takechi T, Okabe H, Ikeda K, et al: Correlations between antitumor activities of fluoropyrimidines and DPD activity in lung tumor xenografts. Oncol Rep 14: 33-39, 2005.

6. Kawahara M, Furuse K, Segawa Y, et al: Phase II study of S-1, a novel oral fluorouracil, in advanced non-small-cell lung cancer. Br J Cancer 85: 939-943, 2001.

7. Suto A, Kubota T, Fukushima M, et al: Antitumor effect of combination of S-1 and docetaxel on the human breast cancer xenograft transplanted into SCID mice. Oncol Rep 15: 1517-1522, 2006.

8. Yoshida K, Hirabayashi N, Takiyama N, et al: Phase I study of combination therapy with S-1 and docetaxel (TXT) for advanced or recurrent gastric cancer. Anticancer Res 24: 1843-1851, 2004.

9. Yoshida K, Ninomiya M, Takakura N, et al: Phase II study of docetaxel and S-1 combination therapy for advanced or recurrent gastric cancer. Clin Cancer Res 12: 3402-3407, 2006.

10. Pfister DG, Johnson DH, Azzoli CG, et al: American Society of Clinical Oncology treatment of unresectable non-small-cell lung cancer guideline: update 2003. J Clin Oncol 22: 330-353, 2004.

11. Azzoli CG, Baker S Jr, Temin S, et al: American Society of Clinical Oncology clinical practice guideline update on chemotherapy for stage IV non-small-cell lung cancer. J Clin Oncol 27: 6251-6266, 2009.

12. Noble J, Ellis PM, Mackay JA, et al: Second-line or subsequent systemic therapy for recurrent or progressive non-small cell lung cancer: a systematic review and practice guideline. J Thorac Oncol 1: 1042-1058, 2006.

13. Takahashi I, Emi Y, Kakeji Y, et al: Phase I study of S-1 and biweekly docetaxel combination chemotherapy for advanced and recurrent gastric cancer. Oncol Rep 15: 849-854, 2006.

14. Atagi S, Kawahara M, Kusunoki Y, et al: Phase I/II study of doxetaxel and S-1 in patients with previously treated non-small cell lung cancer. J Thorac Oncol 3: 1012-1017, 2008.

15. Fidias PM, Dakhil SR, Lyss AP, et al: Phase III study of immediate compared with delayed docetaxel after frontline therapy with gemcitabine plus carboplatin in advanced non-small cell lung cancer. J Clin Oncol 27: 591-598, 2009. 